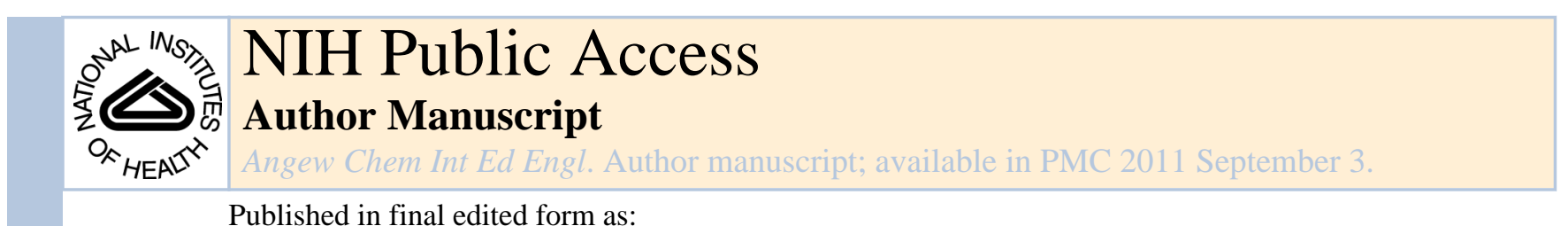

Published in final edited form as:

Angew Chem Int Ed Engl. 2010 September 3; 49(37): 6676-6678. doi:10.1002/anie.201003272.

\title{
Alkyl-Alkyl Suzuki Cross-Couplings of Unactivated Secondary Alkyl Chlorides*
}

\author{
Zhe Lu and Prof. Dr. Gregory C. Fu* \\ Department of Chemistry, Massachusetts Institute of Technology, Cambridge, MA 02139 (USA)
}

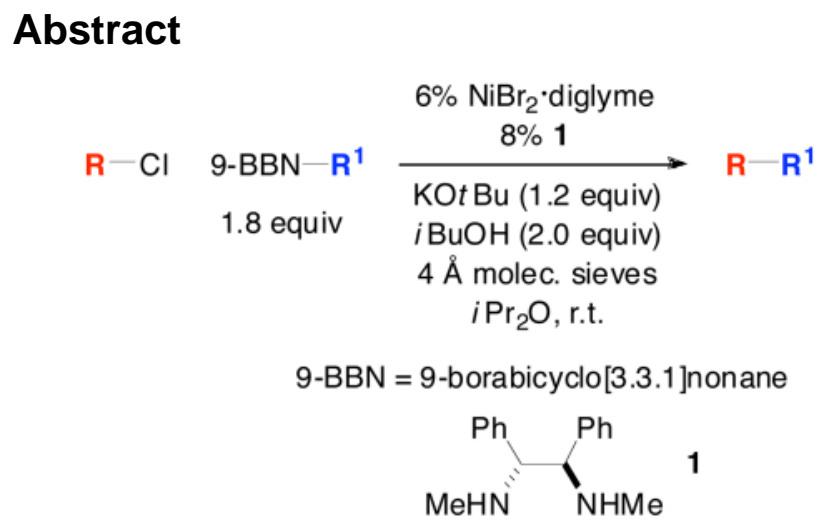

The first method for achieving alkyl-alkyl Suzuki reactions of unactivated secondary alkyl chlorides has been developed. Carbon-carbon bond formation occurs under mild conditions (at room temperature) with the aid of commercially available catalyst components. This method has proved to be versatile: without modification, it can be applied to Suzuki reactions of secondary and primary alkyl bromides and iodides, as well as primary alkyl chlorides. Mechanistic investigations suggest that oxidative addition is not the turnover-limiting step of the catalytic cycle for unactivated secondary alkyl iodides and bromides, whereas it may be (partially) for chlorides.

\section{Keywords}

alkyl halide; boron; catalysis; cross-coupling; nickel

\begin{abstract}
Among cross-coupling processes that form carbon-carbon bonds, the Suzuki reaction is perhaps the most widely used, due to considerations such as functional-group tolerance, the accessibility of organoboron coupling partners, and toxicity issues.[1] Most of the early investigations of Suzuki cross-couplings focused on reactions of aryl and vinyl electrophiles. Of course, the ability to also couple a wide range of alkyl electrophiles would significantly enhance the utility of Suzuki reactions, and advances toward this goal have been described. [2,3] Despite this progress, many classes of alkyl-alkyl Suzuki cross-couplings still have not been achieved, including reactions of unactivated secondary alkyl chlorides.[4,5] In this
\end{abstract}

\footnotetext{
** Support has been provided by the National Institutes of Health (National Institute of General Medical Sciences, grant R01GM62871), Eli Lilly (fellowship to Z.L.), the Martin Family Society of Fellows for Sustainability (fellowship to Z.L.), Merck Research Laboratories, and Novartis. We thank Dr. Bunnai Saito for preliminary studies and Dr. Jeffrey H. Simpson for assistance with NMR spectroscopy.

Fax: (+1) 617-324-3611, gcf@ mit.edu.

Supporting information for this article is available on the WWW under http://**
} 
report, we establish that this family of electrophiles can indeed serve as suitable partners in alkyl-alkyl Suzuki couplings under mild conditions through the use of an appropriate nickel catalyst [Eq. (1)].

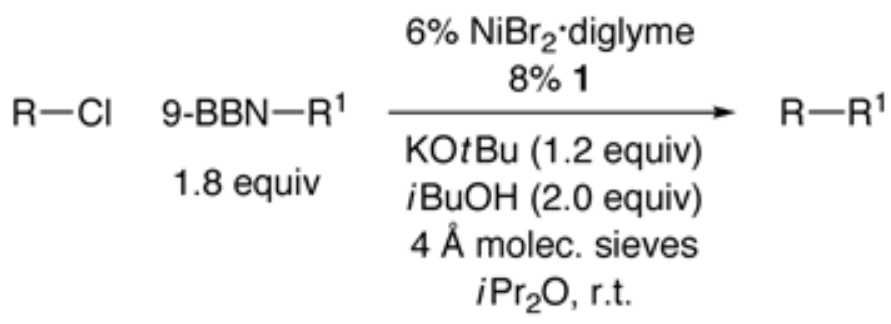

\section{9-BBN = 9-borabicyclo[3.3.1]nonane<smiles>CNC(c1ccccc1)[C@H](NC)c1ccccc1</smiles>

In the case of our method for alkyl-alkyl Suzuki cross-couplings of secondary bromides and iodides,[4a] we observed that the corresponding chlorides are much less reactive. For example, the coupling illustrated in entry 1 of Table 1 proceeds in poor yield (9\%) when that procedure is applied. Nevertheless, by systematically examining the various reaction parameters, we determined that the desired cross-coupling of an unactivated secondary alkyl chloride can in fact be accomplished efficiently at room temperature. Specifically, altering the nickel source (entry $1 \rightarrow$ entry 2 ), the ligand (entry $2 \rightarrow$ entry 3 ), and the solvent (entry $3 \rightarrow$ entry 4 ) led to a method that furnishes the target coupling product in good yield (entry 4 : $86 \%$; all of the catalyst components are commercially available).

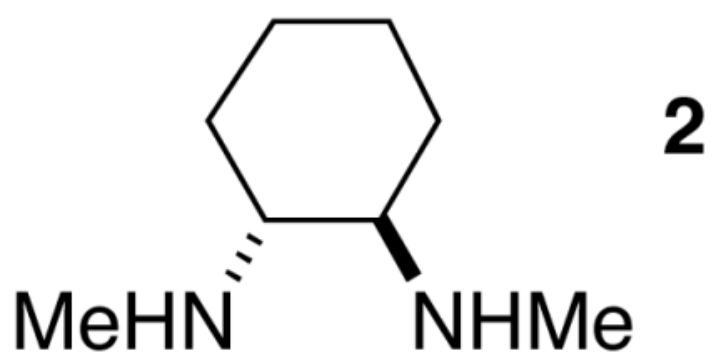

One practical drawback of our previous procedures for alkyl-alkyl Suzuki reactions of secondary bromides/iodides[4] was our observation that cross-couplings that were set up without a glove box proceeded in lower yield than reactions conducted in a glove box. When we attempted to employ the method illustrated in entry 4 of Table 1 without a glove box, we also obtained a diminished yield. We hypothesized that adventitious water might be the culprit, and we therefore added powdered $4 \AA$ molecular sieves to the reaction mixture. Indeed, with this modification, we were able to generate a good yield of the Suzuki coupling product of the secondary chloride without the use of a glove box (83\%). 
As illustrated in Table 2, the scope of this new cross-coupling method is fairly broad.[6] Both cyclic (entries 1-6; including nitrogen and oxygen heterocycles: entries 5 and 6) and acyclic (entries 7-13) secondary alkyl chlorides are suitable substrates. A variety of functional groups are compatible with the reaction conditions, such as alkyl and silyl ethers (entries 2, 6, 8, 9, and 13), alkenes (entry 4), carbamates (entry 5), esters (entries 11 and 12), and acetals (entries 12 and 13).

We were pleased to determine that this method for alkyl-alkyl Suzuki cross-couplings, which we optimized for a new family of substrates (unactivated secondary alkyl chlorides), can be applied without modification to an array of reaction partners. Thus, secondary alkyl bromides and iodides, both cyclic (including heterocyclic) and acyclic, are suitable electrophiles (Table 3, entries 1-8).[7] Furthermore, primary chlorides, bromides, and iodides undergo Suzuki coupling in good yield (entries 9-11).

We have examined the relative reactivity of cyclohexyl halides in Suzuki cross-couplings with an alkylborane under this set of conditions [Eq. (2)]. The iodide and the bromide couple at comparable rates, whereas the chloride reacts more slowly.

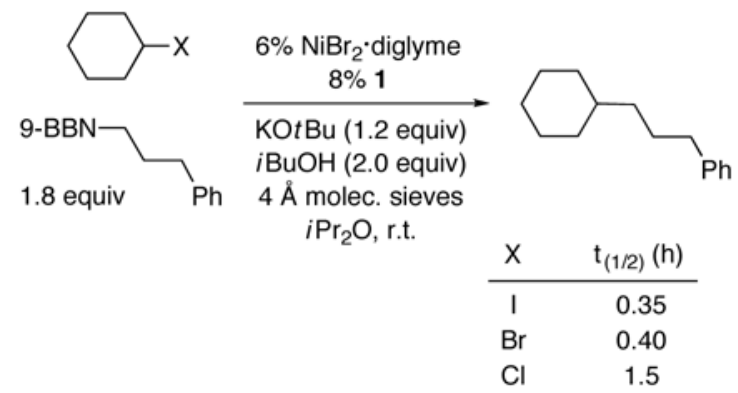

Interestingly, in competition experiments between pairs of cyclohexyl electrophiles, the catalyst differentiates effectively between halides [Eq. (3)].[8] Collectively, these data are consistent with oxidative addition not being the turnover-limiting step of the catalytic cycle in the case of cyclohexyl iodide and bromide.[9]

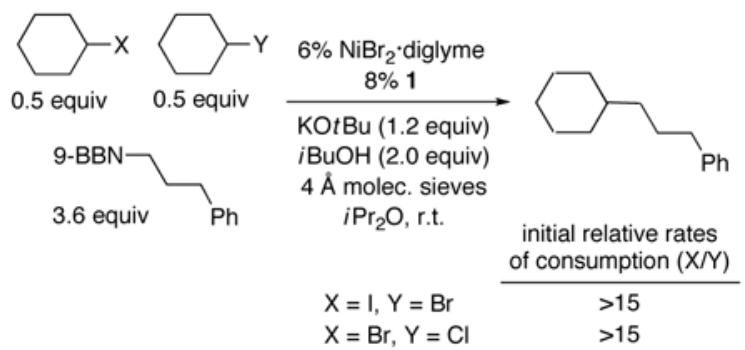

We have determined that, for the reaction of cyclohexyl bromide, the rate law is first order in the catalyst $\left(\mathrm{NiBr}_{2} \bullet\right.$ diglyme/ligand $\left.\mathbf{1}\right)$, first order in the alkylborane, and zeroth order in the electrophile. These data are also consistent with oxidative addition not being the turnover-limiting step for this Suzuki reaction of cyclohexyl bromide. In contrast, for 
cyclohexyl chloride, the rate of cross-coupling is dependent on the concentration of the electrophile.

In conclusion, we have developed the first method for achieving alkyl-alkyl Suzuki reactions of unactivated secondary alkyl chlorides. Carbon-carbon bond formation occurs under mild conditions (at room temperature) with the aid of commercially available catalyst components. This method, although developed for cross-couplings of unactivated secondary chlorides, has proved to be versatile: without modification, it can be applied to Suzuki reactions of secondary and primary alkyl bromides and iodides, as well as primary alkyl chlorides. Mechanistic investigations suggest that oxidative addition is not the turnoverlimiting step of the catalytic cycle for unactivated secondary alkyl iodides and bromides, whereas it may be (partially) for chlorides. Additional mechanistic and catalyst-development studies of a wide array of alkyl-alkyl cross-couplings are underway.

\section{Supplementary Material}

Refer to Web version on PubMed Central for supplementary material.

\section{References}

1. For leading references, see: a) de Meijere, A.; Diederich, F., editors. Metal-Catalyzed CrossCoupling Reactions. Wiley-VCH; New York: 2004. b) Negishi, E-i, editor. Handbook of Organopalladium Chemistry for Organic Synthesis. Wiley Interscience; New York: 2002. c) Miyaura, N., editor. Topics in Current Chemistry Series. Vol. 219. Springer-Verlag; New York: 2002. Cross-Coupling Reactions: A Practical Guide.

2. For a pioneering study of Suzuki reactions of primary alkyl electrophiles, see: a) Ishiyama T, Abe S, Miyaura N, Suzuki A. Chem Lett. 1992:691-694.For a review of cross-couplings of secondary alkyl electrophiles, see: b) Rudolph A, Lautens M. Angew Chem, Int Ed. 2009; 48:2656-2670.

3. For examples of applications of alkyl-alkyl Suzuki cross-couplings in the total synthesis of natural products, see: a) Keaton KA, Phillips AJ. Org Lett. 2007; 9:2717-2719. [PubMed: 17559220] b) Griggs ND, Phillips AJ. Org Lett. 2008; 10:4955-4957. [PubMed: 18844364]

4. For alkyl-alkyl Suzuki reactions of unactivated secondary alkyl bromides and/or iodides, see: a) Saito B, Fu GC. J Am Chem Soc. 2007; 129:9602-9603. [PubMed: 17628067] b) Saito B, Fu GC. J Am Chem Soc. 2008; 130:6694-6695. [PubMed: 18447357]

5. For alkyl-aryl Suzuki reactions of unactivated secondary alkyl iodides, bromides, and chlorides at $60{ }^{\circ} \mathrm{C}$, see: González-Bobes F, Fu GC. J Am Chem Soc. 2006; 128:5360-5361. [PubMed: 16620105] See also: Zhou J, Fu GC. J Am Chem Soc. 2004; 126:1340-1341. [PubMed: 14759182]

6. Notes: a) In the absence of $\mathrm{NiBr}_{2} \bullet$ diglyme or ligand $\mathbf{1}$, essentially none of the desired product is formed $(<1 \%)$; b) On a gram scale, the Suzuki reaction illustrated in entry 1 of Table 2 proceeds in $79 \%$ yield (isolated yield, average of two experiments); c) Under our standard conditions, secondary alkylboranes, primary alkylboronate esters, primary alkylboronic acids, and primary alkyltrifluoroborates do not cross-couple in good yield; d) By ${ }^{11} \mathrm{~B}$ NMR spectroscopy, we have determined that the potassium alkoxide reacts with the alkyl-9-BBN reagent to form a tetravalent boron complex. In addition to activating the trialkylborane, this interaction attenuates the Brönsted basicity of the reaction mixture; e) On a 1-mmol scale in a microwave $\left(60{ }^{\circ} \mathrm{C}\right.$ for 3.5 hours), the cross-coupling depicted in entry 1 of Table 2 proceeds in $74 \%$ yield; f) Compared with $i \operatorname{Pr}_{2} \mathrm{O}, t$ $\mathrm{BuOMe}$ and $\mathrm{Et}_{2} \mathrm{O}$ furnish slightly lower yields, whereas THF provides a greatly diminished yield.

7. Under our standard conditions, attempts to cross-couple an unactivated tertiary chloride, bromide, and iodide led to essentially none of the desired products.

8. High selectivity $(\mathrm{I}>\mathrm{Br}>\mathrm{Cl})$ is often observed in oxidative additions/abstractions that proceed via an inner-sphere electron-transfer pathway. For example, see: Scott SL, Espenson JH, Zhu Z. J Am Chem Soc. 1993; 115:1789-1797.

9. For mechanistic proposals for nickel-catalyzed Negishi reactions of alkyl electrophiles, see: a) Jones GD, Martin JL, McFarland C, Allen OR, Hall RE, Haley AD, Brandon RJ, Konovalova T, 
Desrochers PJ, Pulay P, Vicic DA. J Am Chem Soc. 2006; 128:13175-13183. [PubMed: 17017797]

b) Lin X, Phillips DL. J Org Chem. 2008; 73:3680-3688. [PubMed: 18410144] 
Table 1

Effect of reaction parameters on an alkyl-alkyl Suzuki cross-coupling of an unactivated secondary alkyl chloride.

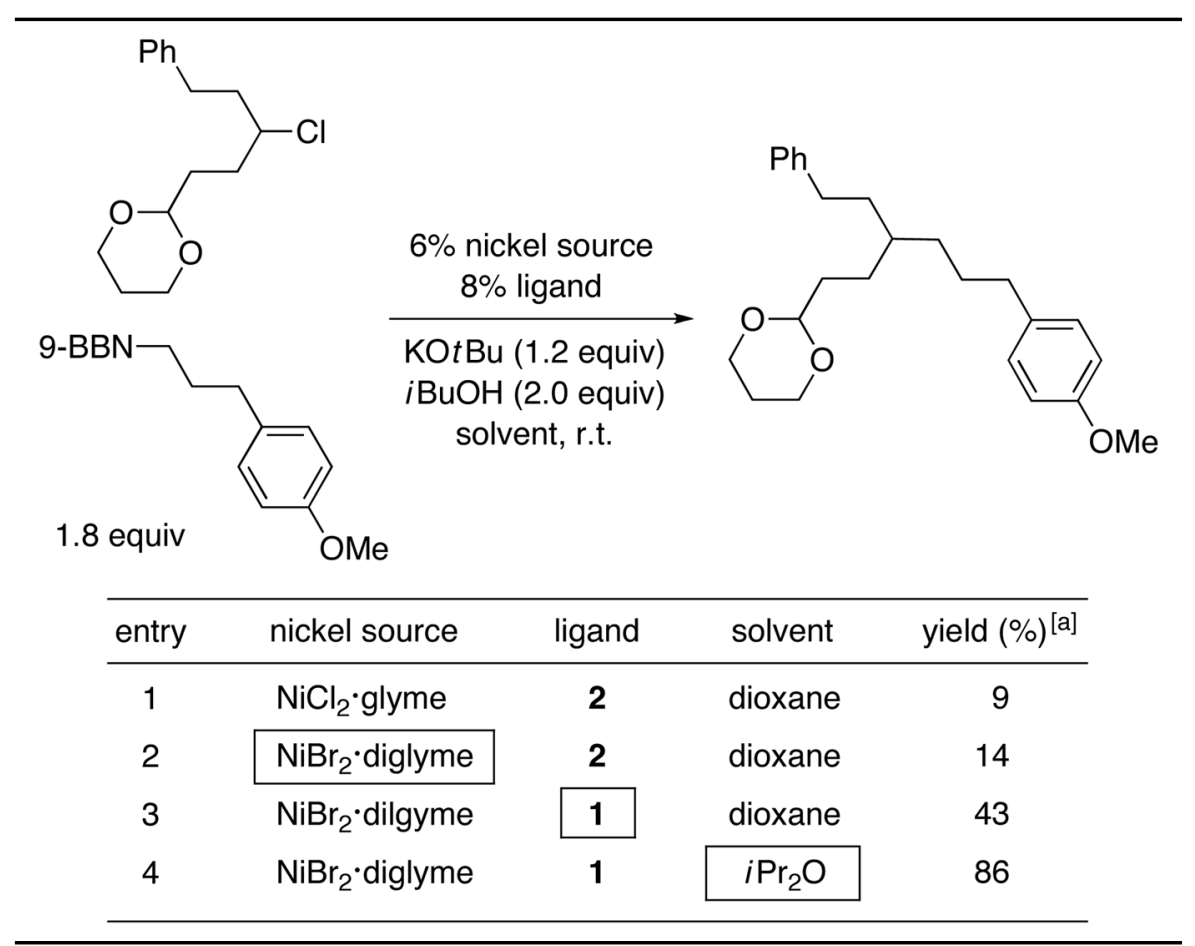

${ }^{[a]}$ Determined by GC versus a calibrated internal standard (average of two experiments). 
Table 2

Alkyl-alkyl Suzuki cross-couplings of unactivated secondary alkyl chlorides [for the reaction conditions, see Eq. (1)].

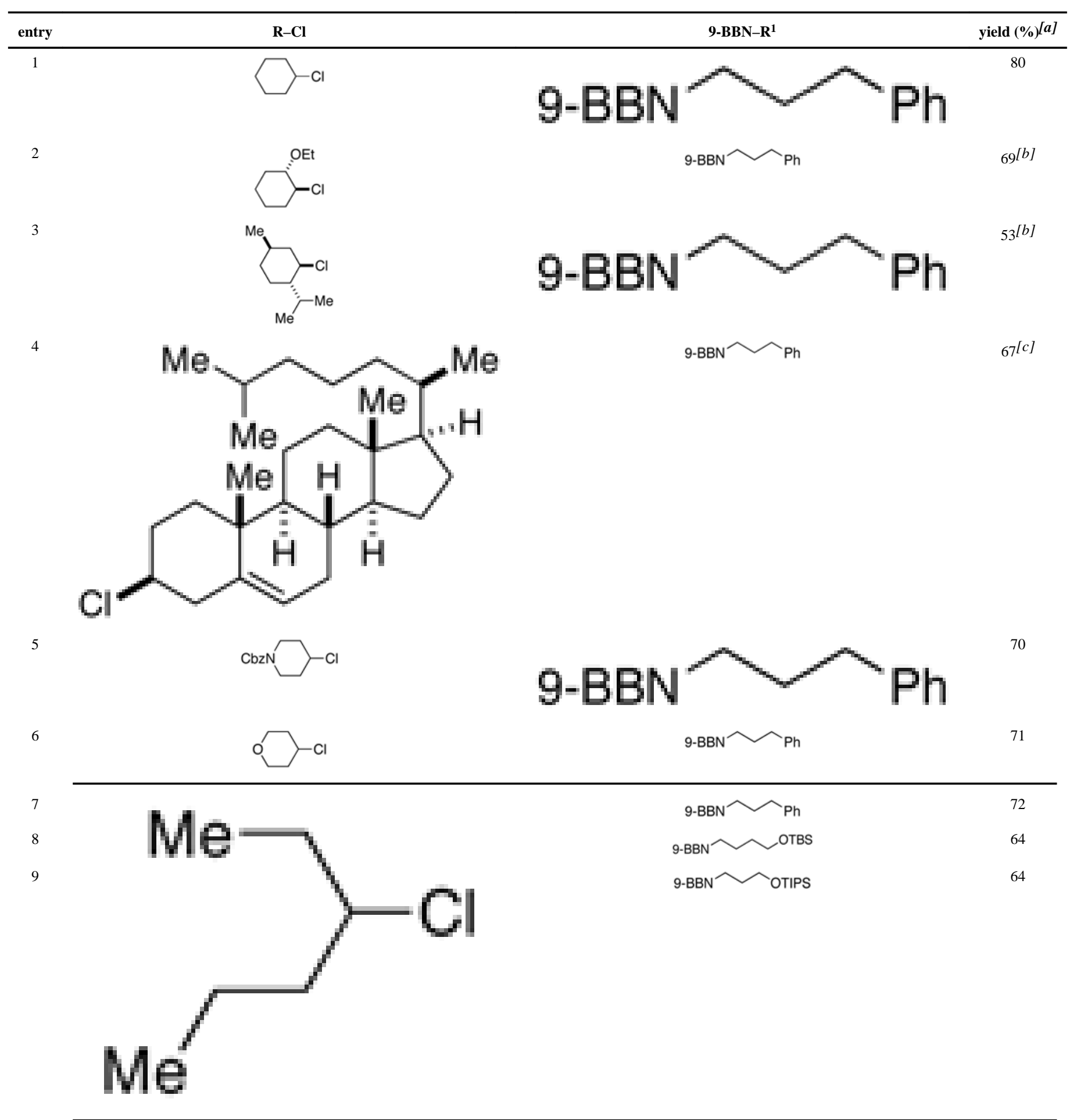




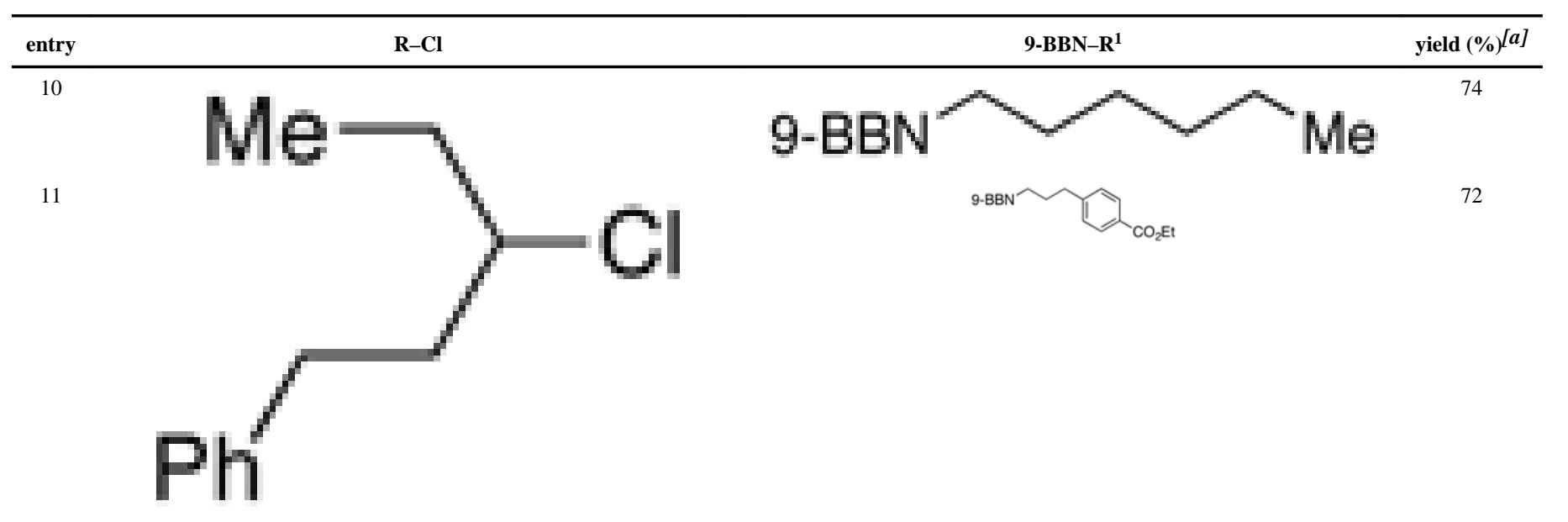

12

13

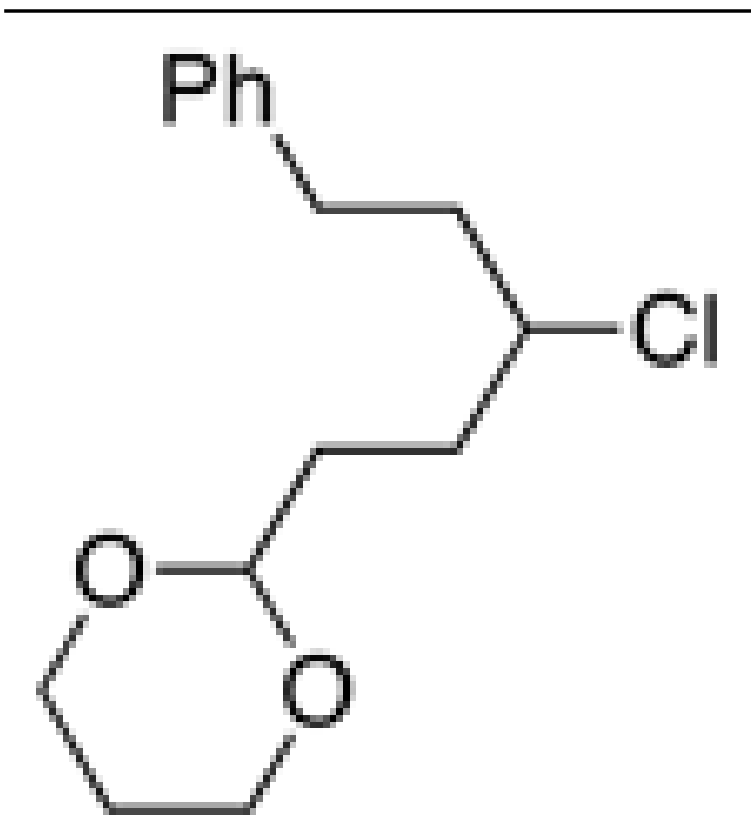

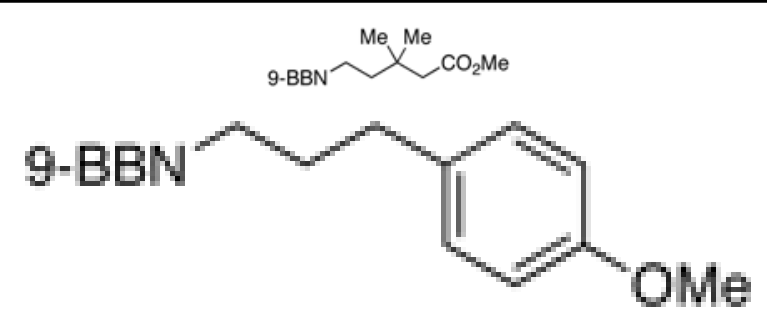

${ }^{[a]}$ Isolated yield (average of two experiments).

${ }^{[b]}$ Diastereoselectivity: >20:1 trans:cis (relative to the proximal substituent).

${ }^{[c]}$ Diastereoselectivity: 2:1 $\beta: \alpha$. 
Table 3

Alkyl-alkyl Suzuki cross-couplings of unactivated alkyl halides [for the reaction conditions, see Eq. (1)].

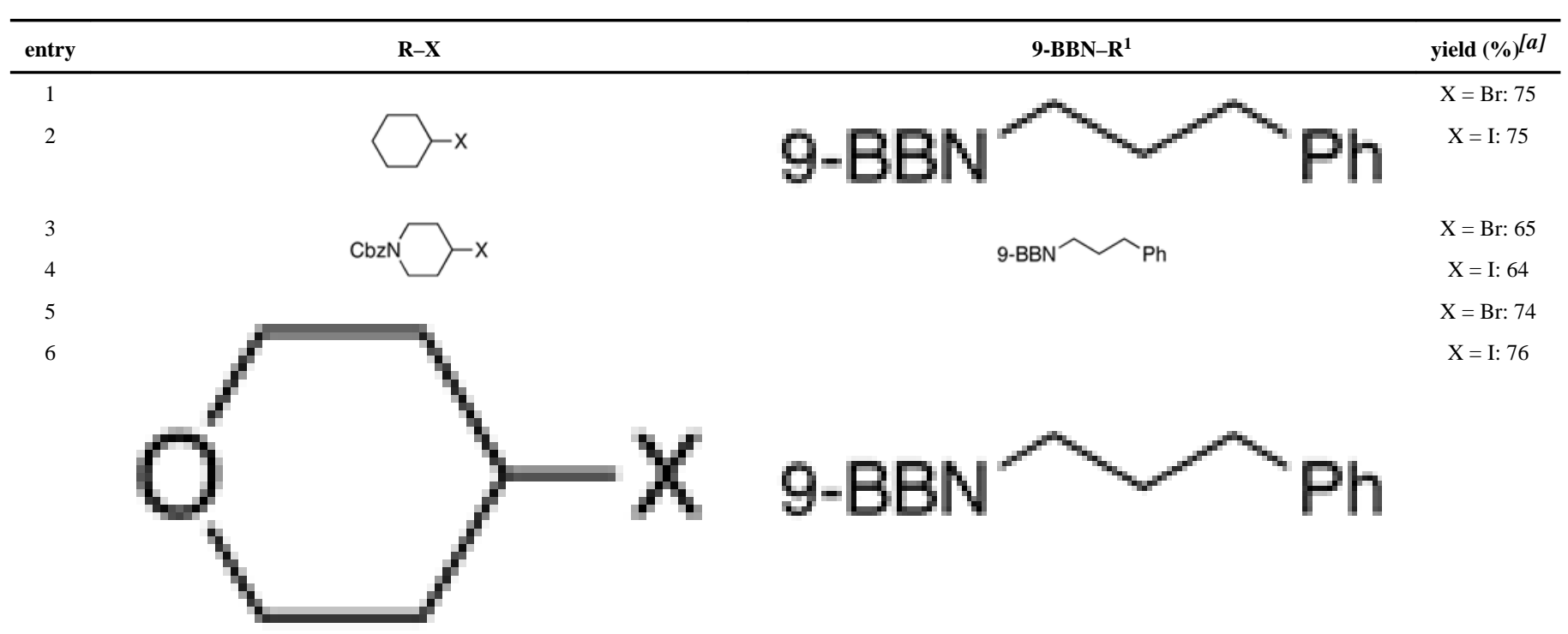

7

8

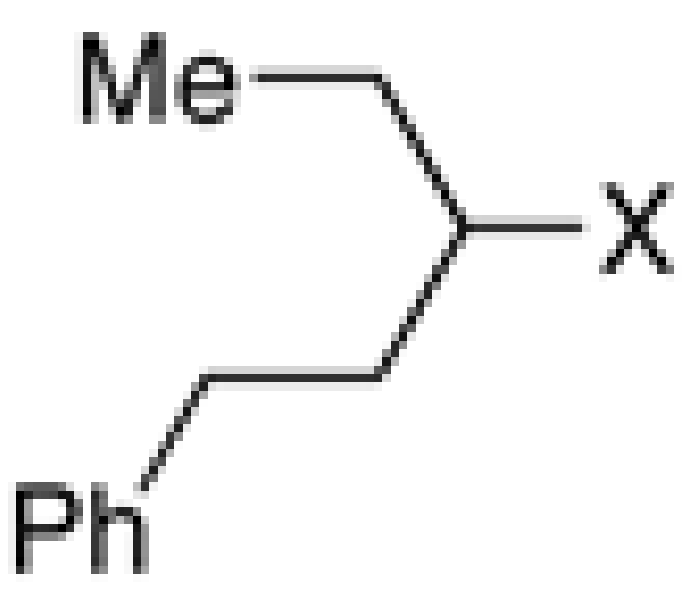

$$
\mathrm{TsO}+{\sqrt{1_{4}}}^{\mathrm{Cl}}
$$

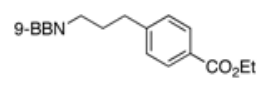

10

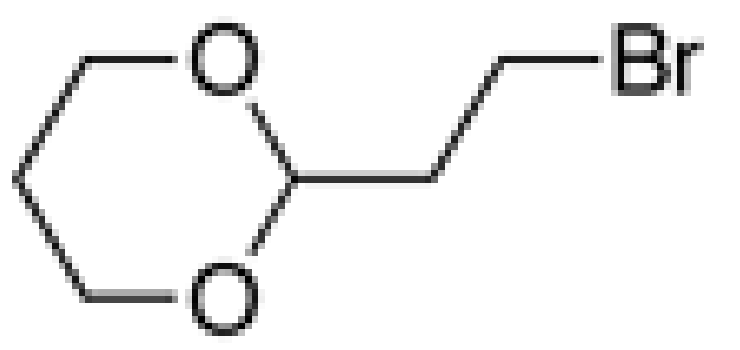

9-BBN

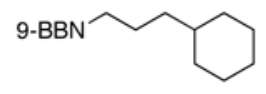

$\mathrm{X}=\mathrm{Br}: 75$

$\mathrm{X}=\mathrm{I}: 76$

63

70

78 
${ }^{[a]}$ Isolated yield (average of two experiments). 